\title{
Introducing Foldit Education Mode
}

To the Editor - In its twelve years online, Foldit has become a frequently used teaching tool for biochemistry at the college level, despite not providing premade content specifically for education. Here we introduce Foldit Education Mode, in which students proceed through a self-guided tutorial of standard concepts in protein biochemistry via a series of interactive three-dimensional biochemistry puzzles. Education Mode could be used independently or as a central component of a science class. With many classrooms staying in remote mode this autumn, Foldit Education Mode offers a unique and free online option for classes worldwide and is available for download at our new educator's page: https://fold.it/educator.

Foldit is a free citizen science biochemistry computer game ${ }^{1}$. In the most basic Foldit puzzles, players try to fold a protein into its proper three-dimensional structure, starting from an unfolded peptide. The players' structures are immediately scored using the Rosetta force field ${ }^{2}$, with the players collaborating and competing to attain the best-scoring protein structure. The concept is that, if hundreds or thousands of players all work to create a protein structure, there is a higher chance that one of them may find the correct fold.

The interactive nature of Foldit and its accessibility to non-scientist audiences quickly attracted the interest of educators ${ }^{3-6}$, despite Foldit not providing material that is explicitly tailored for science education. We have recently introduced Custom Contests ${ }^{7}$, a feature that allows educators to create, administer and grade their own Foldit puzzles to fit their curriculum, but Custom Contests do not provide pre-made content to teach standard topics in biochemistry.

The need for online learning opportunities has vastly increased in recent months due to the COVID-19 pandemic, and we have responded by creating Foldit Education Mode. Currently, Education Mode consists of 29 puzzles split into 9 tiers that walk the student through a standard set of topics in biochemistry education (Fig. 1a), including atomic interactions, the hydrophobic effect, amino acids, primary structure, secondary structure, tertiary structure, protein folding pathways and ligand binding. Many of the puzzles start by allowing the student to explore the puzzle as a sandbox, thus becoming familiar with it before starting the tutorial. In each puzzle, students click through a tutorial on the topic that guides them through the conceptual basis of the puzzle (Fig. 1b). Then, students solve the puzzle by achieving a prespecified Foldit score. In many levels, this score is modified to provide a bonus that rewards specific outcomes, such as rewarding the creation of hydrogen bonds in a puzzle that judges $\beta$-sheet formation. Along the way, students learn Foldit tools that will allow them to complete more advanced puzzles. These are a mixture of protein design puzzles (Fig. 1c), folding puzzles (Fig. 1d) and sequence alignment a

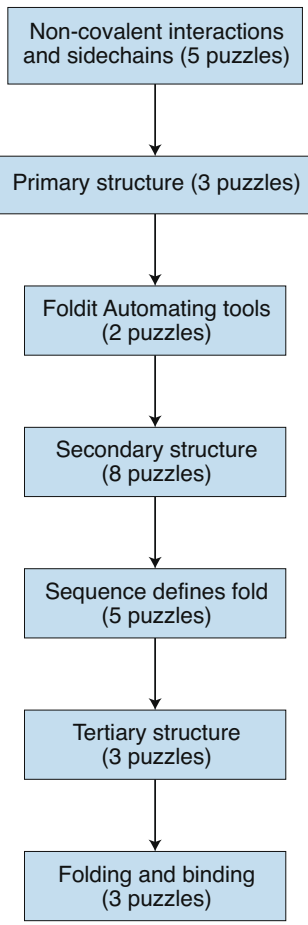

b

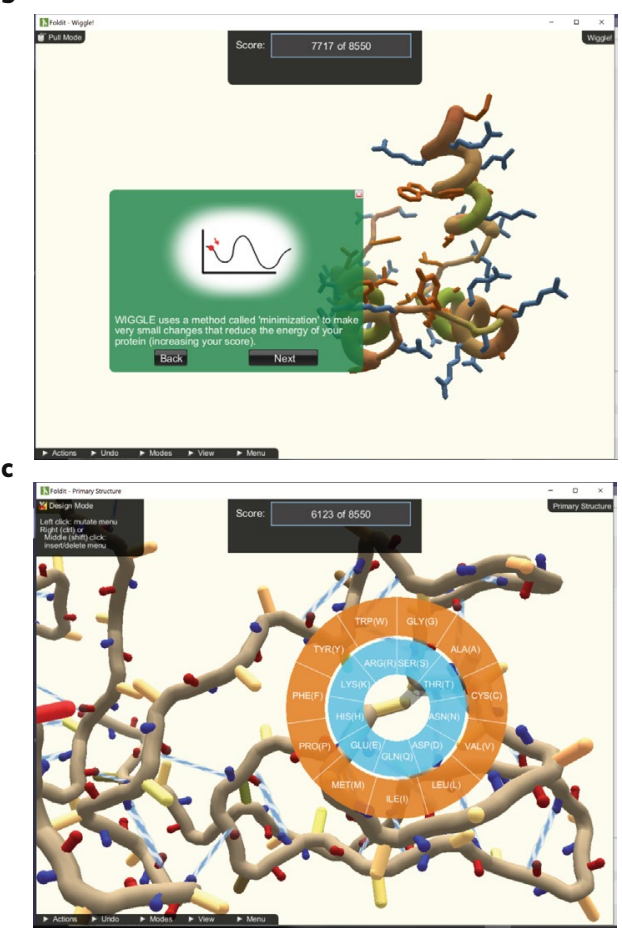

d

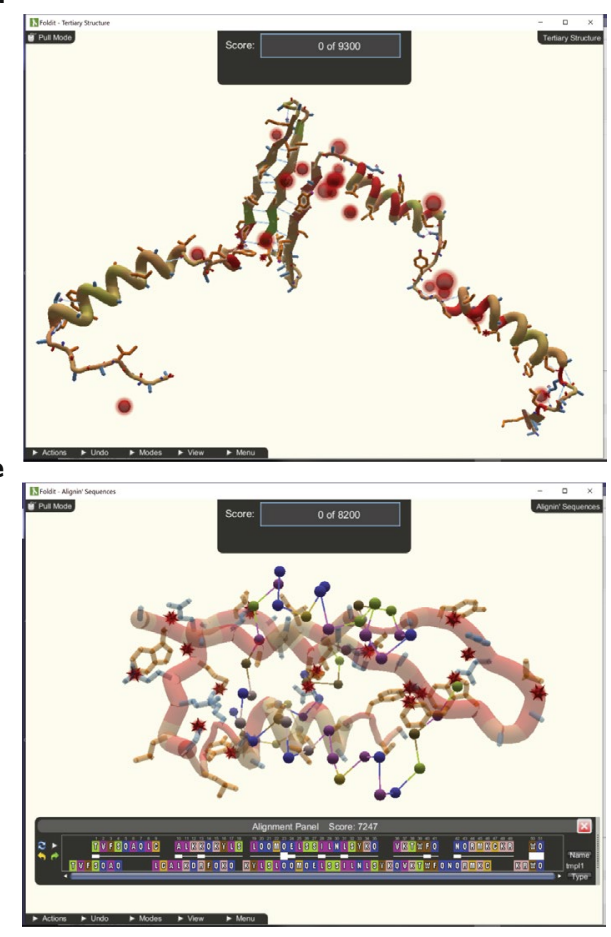

Fig. 1 | Foldit Education Mode. a, A flowchart depicting the current topics and number of puzzles within Foldit Education Mode. b, Wiggle puzzle, showing a tutorial bubble example. Students click back or next to proceed through the tutorials within each puzzle. c, An example of a protein design puzzle, "Primary Structure," in which students are asked to choose amino acids that optimize the protein's stability. d, An example of a protein folding puzzle, "Tertiary Structure," in which students are given a protein with preformed secondary structure and are asked to fold it into a stable tertiary structure. e, An example of a sequence alignment puzzle. In this puzzle, "Alignin' Sequences," students are asked to align the sequence of a protein to that of a protein of known structure to enable structural threading. 
puzzles (Fig. 1e), which are used to teach different topics.

Although the tutorial is self-guided and can be used by anyone interested in learning biochemistry, it was specifically created for class use. Instructors can choose to skip puzzles as needed and can supplement with puzzles from the standard Foldit tutorials if desired. Several of the puzzles have already been used in class, with accompanying written assignments that can be downloaded at our educator's webpage (https://fold.it/educator). Students are also able to use the in-game chat function to discuss their puzzles in real time with other students in their class or with their instructor.

Some important notes: students are currently unable to turn in their puzzles for grading through the Foldit client, and the instructor does not have access to their solutions. These features are available in Custom Contests; thus, for larger and more involved puzzles with accompanying written assignments (for example, the "Primary Structure" and "Tertiary Structure" puzzles), instructors may consider skipping the version in Education Mode and downloading a Custom Contest version of the puzzle instead. Moreover, we note that Education Mode is a different release from standard Foldit, and playing standard science puzzles will require switching to the main release of Foldit.

Finally, we are making this announcement before Education Mode has undergone stringent educational testing, due to the urgent need for such tools right now. We welcome all feedback on how to improve the Education Mode.

\section{Josh Aaron Miller (D)1, Firas Khatib (D), Haley Hammond ${ }^{3}$, Seth Cooper ${ }^{1}$ and Scott Horowitz (D) ${ }^{3 凶}$}

${ }^{1}$ Khoury College of Computer Sciences, Northeastern University, Boston, MA, USA. ${ }^{2}$ Department of Computer and Information Science, University of Massachusetts Dartmouth, Dartmouth, MA, USA.
${ }^{3}$ Department of Chemistry \& Biochemistry and the Knoebel Institute for Healthy Aging, University of Denver, Denver, CO, USA.

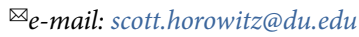

Published online: 17 August 2020

https://doi.org/10.1038/s41594-020-0485-6

References

1. Cooper, S. et al. Nature 466, 756-760 (2010).

2. Alford, R. F. et al. J. Chem. Theory Comput. 13, 3031-3048 (2017)

3. Farley, P. C. Biochem. Mol. Biol. Educ. 41, 56-57 (2013).

4. Franco, J. J. Chem. Educ. 89, 1543-1546 (2012).

5. Stockman, B. J. et al. J. Chem. Educ. 91, 451-454 (2014).

6. Achterman, R. R. J. Microbiol. Biol. Educ. 20, 20.3.63 (2019).

7. Dsilva, L. et al. Biochem. Mol. Biol. Educ. 47, 133-139 (2019).

\section{Acknowledgements}

We thank Kimberly Cortes, Ashley Vater and Irina Makarevitch for their prerelease input and Jeff Flatten, Zoran Popović, Henry Solberg, Rocco Moretti, David Baker and Jens Meiler for aid with Foldit support.

This work was supported by NIH R00 GM120388.

Competing interests

The authors declare no competing interests. 